\title{
Instituto de Química de la UnAM: catalizando la química con paso firme
}

\author{
Tomás Guerrero, * José G. López-Cortés*
}

RESUMEN: El Instituto de Química de la Universidad Nacional Autónoma de México (IQ-UNAM), a sus 75 años de existencia, se ha forjado como una institución sólida y de prestigio internacional. Recientemente, una de las líneas de investigación con mayor impacto en el IQ-UNAM, por su trascendencia en todas las áreas de la química, es la catálisis. En el IQ se cuenta con la infraestructura necesaria para llevar a cabo investigación básica y aplicada en el área de la catálisis homogénea. La participación de la institución en diversos programas de posgrado permite la generación de productos de investigación, proyectos en colaboración con la industria, la generación de conocimiento indispensable para la química y para nuestro país, así como la formación de recursos humanos de alta calidad. Dentro del IQ, un nutrido grupo de investigadores exploran y enriquecen líneas sobre catálisis mediada por metales de transición, el diseño de ligantes y auxiliares quirales, y organocatálisis. La capacidad de vinculación que ofrece el IQ con otras instituciones a nivel mundial lo convierten en la punta de lanza para nuevos descubrimientos en catálisis, transformándolo en uno de los mejores sitios en el mundo para desarrollar esta área de la química.

PALABRAS CLAVE: IQ-UNAM, catálisis, organocatálisis, ligantes, metales de transición.

ABSTRACT: The Institute of Chemistry of the National Autonomous University of Mexico (Spanish acronym: IQ-UNAM), at his $75^{\text {th }}$ anniversary is known around the world as an important and solid institution. Catalysis is outlined as a very strong research field because of their importance in every area of applied chemistry. The development and understanding of several catalytic systems are encouraged among several researchers. IQ is fully equipped with cutting edge technology suitable for basic and applied research in catalysis and related fields. This capability and the strong presence in several graduate programs have an impact in the number of high quality publications, strong collaboration with industry, development of chemistry and high quality of human resources. A strong group of researchers explores and develop transition metal mediated process, design of chiral ligands and organocatalysis. The strong relations with other institutions worldwide makes the IQ the tip of the sword to achieve new discoveries in catalysis and one of the best places in the world to make chemistry.

KEYWORDS: IQ-UNAM, catalysis, organocatalysis, binders, transition metals.

\section{Introducción}

El Instituto de Química de la Universidad Nacional Autónoma de México (figura 1) fue inaugurado el 5 de abril de 1941 en las instalaciones de Tacuba de la

Recibido: 23 de enero de 2017. Aceptado: 25 de marzo de 2017.

*Instituto de Química, Universidad Nacional Autónoma de México, Circuito exterior, Ciudad Universitaria, C.P. 04360, Cd. Mx., México.

Correspondencia: (jglcvdw@unam.mx); Fax: +52 55 56162203; Tel: +52 5556224513. 
Mundo Nano | CATÁLISIS EN MÉXICo | www.mundonano.unam.mx

10(18), enero-junio 2017 | Dol: 10.22201/ceiich.24485691e.2017.18.60033

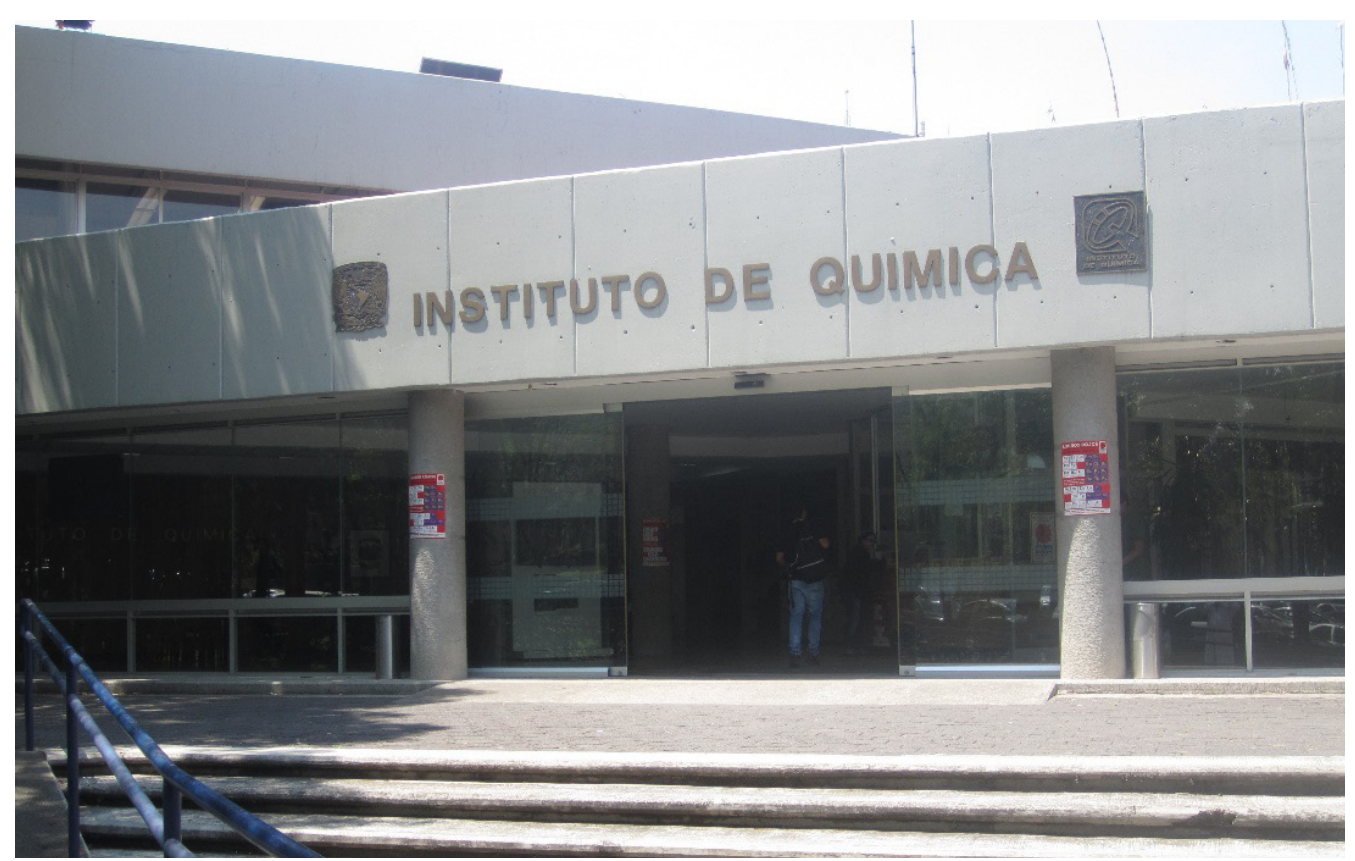

FIGURA 1. Instalaciones del Instituto de Química de la Universidad Nacional Autónoma de México, Ciudad Universitaria, 2017.

antigua Escuela Nacional de Ciencias Químicas y forma parte del Subsistema de la Investigación Científica. Su misión inicial fue organizar la investigación científica en el campo de la química en México con la finalidad de institucionalizarla, de tal manera el instituto generó el nombramiento de Investigador de Tiempo Completo siendo Alberto Sandoval y Fernando Orozco los primeros académicos en obtenerlo (figura 2).

Así el instituto participó de momentos clave en el desarrollo de la industria química del país, y en los primeros momentos de la expropiación petrolera que coinciden con su fundación, se desarrolló la síntesis industrial del tetraetilo de plomo, lo cual permitió la comercialización de las gasolinas en México; posteriormente los estudios de la alcalinidad del lago de Texcoco llevaron a la fundación de la empresa Sosa Texcoco.

Un investigador del instituto, Luis E. Miramontes se dedicó a la síntesis y caracterización de compuestos esteroidales con actividad biológica [1], esta línea de investigación generó un fuerte vínculo con la empresa Syntex, dando origen a un intenso periodo de investigación en el área de la química medicinal, culminando con la síntesis del primer anticonceptivo oral, compuesto esteroidal que revolucionó a la sociedad a nivel mundial.

En sus inicios, el IQ se caracterizó por el estudio de productos naturales, entre otros logros, se puede citar el aislamiento y caracterización de 
los primeros sesterterpenos [2], el estudio de los mecanismos de reacción dienona-fenol, así como el aislamiento y caracterización de la biodiversidad estructural que llevaron al instituto a ser líder mundial en el estudio de lactonas sesquiterpénicas. Siendo referente de vanguardia tecnológica en la elucidación estructural de compuestos químicos, es el lugar donde se adquiere el primer espectrómetro de resonancia magnética nuclear y el primer difractómetro de rayos $\mathrm{X}$ del país, con el tiempo el IQ se vuelve sede del laboratorio nacional de macromoléculas, logrando la obtención de la primera estructura en estado sólido de una proteína en Latinoamérica, la heveína [3].

Al día de hoy, el Instituto de Química cuenta con liderazgos científicos bien establecidos, distribuidos en todos sus departamentos académicos que abarcan todas las áreas de la química. Cuenta con los Departamentos de Productos Naturales y de Química Orgánica que son, por excelencia, departamentos fundadores de la institución así como con un Departamento de Química Inorgánica, sólido y con una visión amplia de las necesidades tecnológicas y los retos que en materia de química, catálisis y procesos deben ser resueltos en los años por venir.

Para dar respuesta a estos retos, el IQ tiene 105 miembros de su personal académico, distribuido entre investigadores y técnicos académicos, cada uno en las diferentes categorías y niveles tanto en el Programa de Primas al Desempeño del Personal Académico de Tiempo Completo (PRIDE) como del Sis-

FIGURA 2. Primeros investigadores con nombramiento en el IQ. Izquierda: Dr. Fernando Orozco, primer director del Instituto de Química; derecha: Dr. Alberto Sandoval Landazúri, primer estudiante mexicano en recibir el grado de doctor en esta institución.
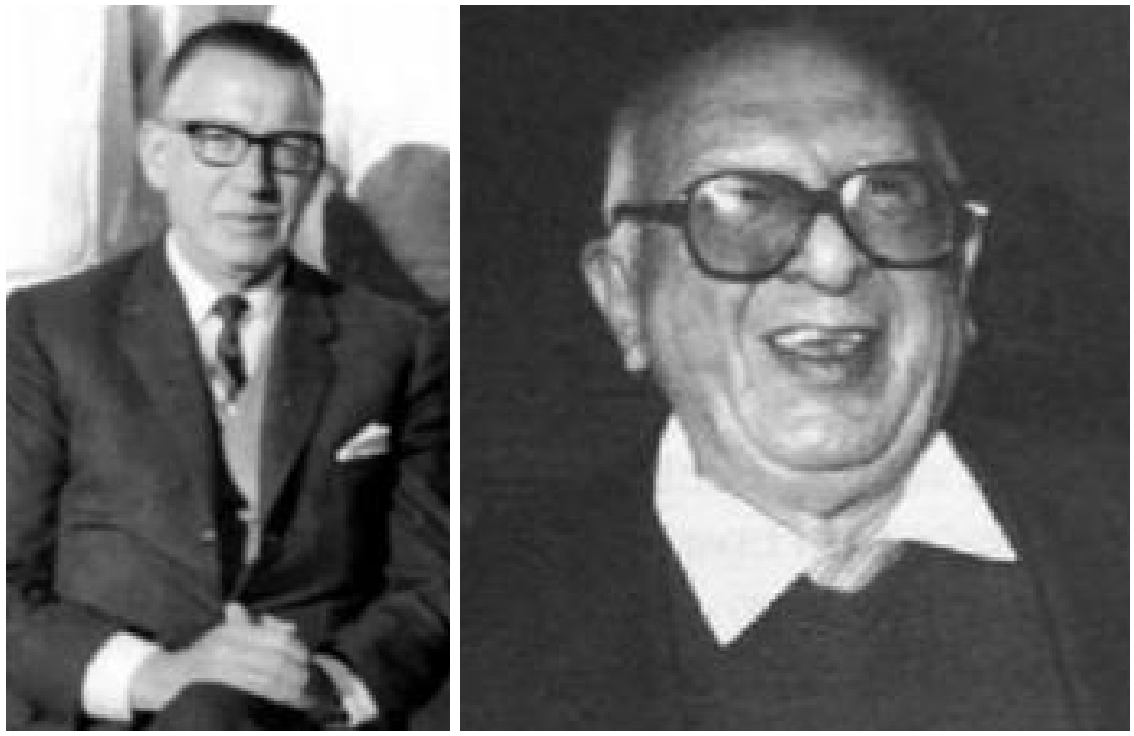
Mundo Nano | CATÁLISIS EN MÉxICo | www.mundonano.unam.mx

10(18), enero-junio 2017 | DOI: 10.22201/ceiich.24485691e.2017.18.60033

tema Nacional de Investigadores (SNI), por ejemplo, basta recalcar que el $42 \%$ del personal académico posee la distinción de investigador nacional en los niveles II y III que confiere el Consejo Nacional de Ciencia y Tecnología (CONACyT), lo que muestra la excelente calidad de la investigación que se realiza en el instituto [4].

\section{Infraestructura}

Para el correcto desarrollo de sus funciones y en aras de garantizar una investigación de alta calidad el instituto cuenta con el más sofisticado equipamiento: equipo para pruebas biológicas completamente automatizado, un nuevo equipo de difracción de rayos X, microscopio de FT-IR, IR, polarimetría, UV-vis y fluorescencia; los servicios analíticos del Instituto de Química se encuentran certificados de acuerdo con la norma Iso 9001:2008 por el Instituto Mexicano de Normalización y Certificación.

Específicamente para el área de catálisis, el IQ cuenta con un laboratorio completo de cromatografía, tanto de gases, como de líquidos, también es factible acoplar esos sistemas a espectrometría de masas, lo que permite la separación, identificación y cuantificación de mezclas complejas (figura 3).

FIGURA 3. Equipos del laboratorio de cromatografía del IQ, a la izquierda se observa un equipo de HPLC acoplado a UV-vis, a la derecha, un equipo Agilent 1200 acoplado a un espectrómetro de masas.
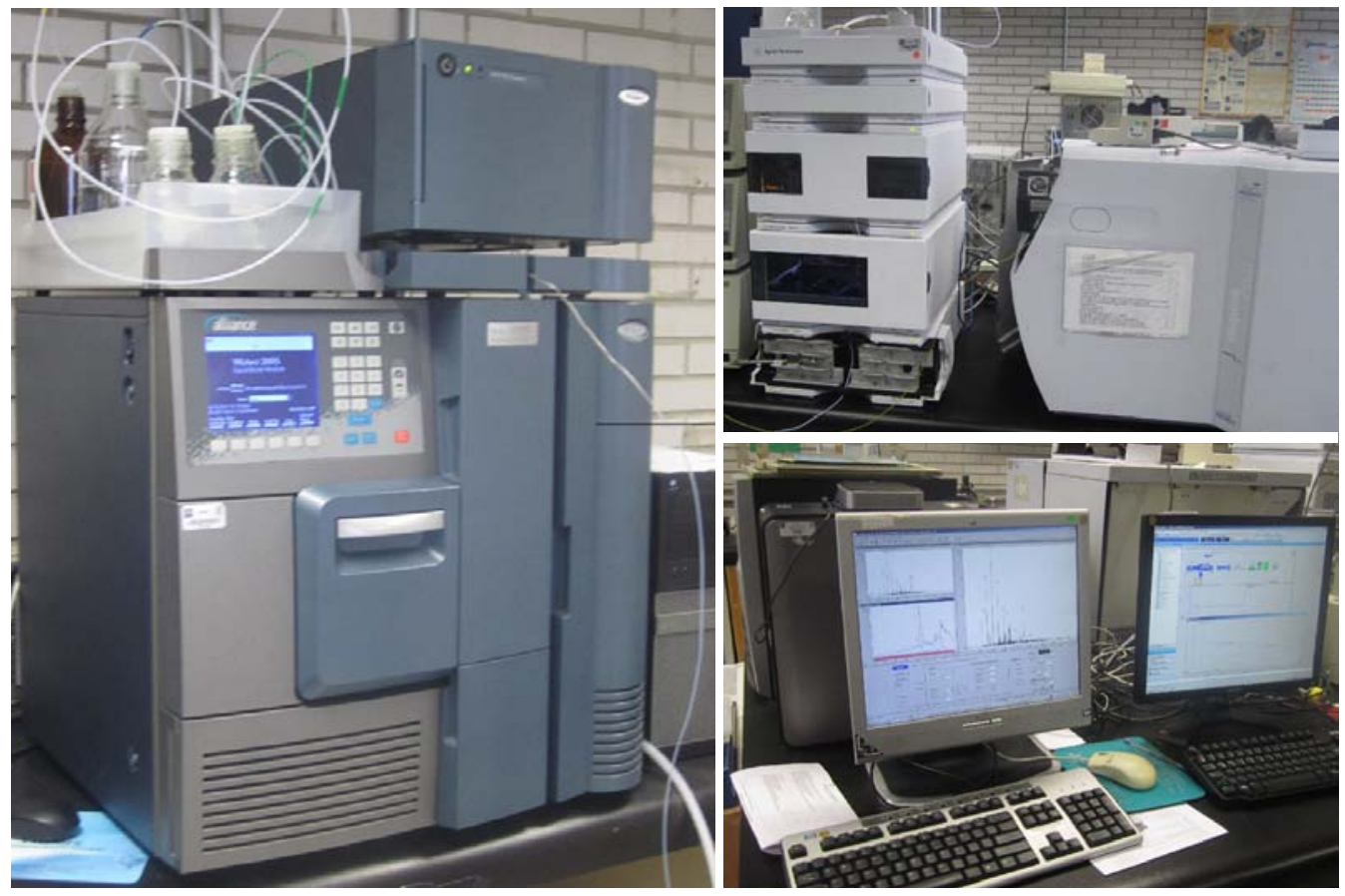

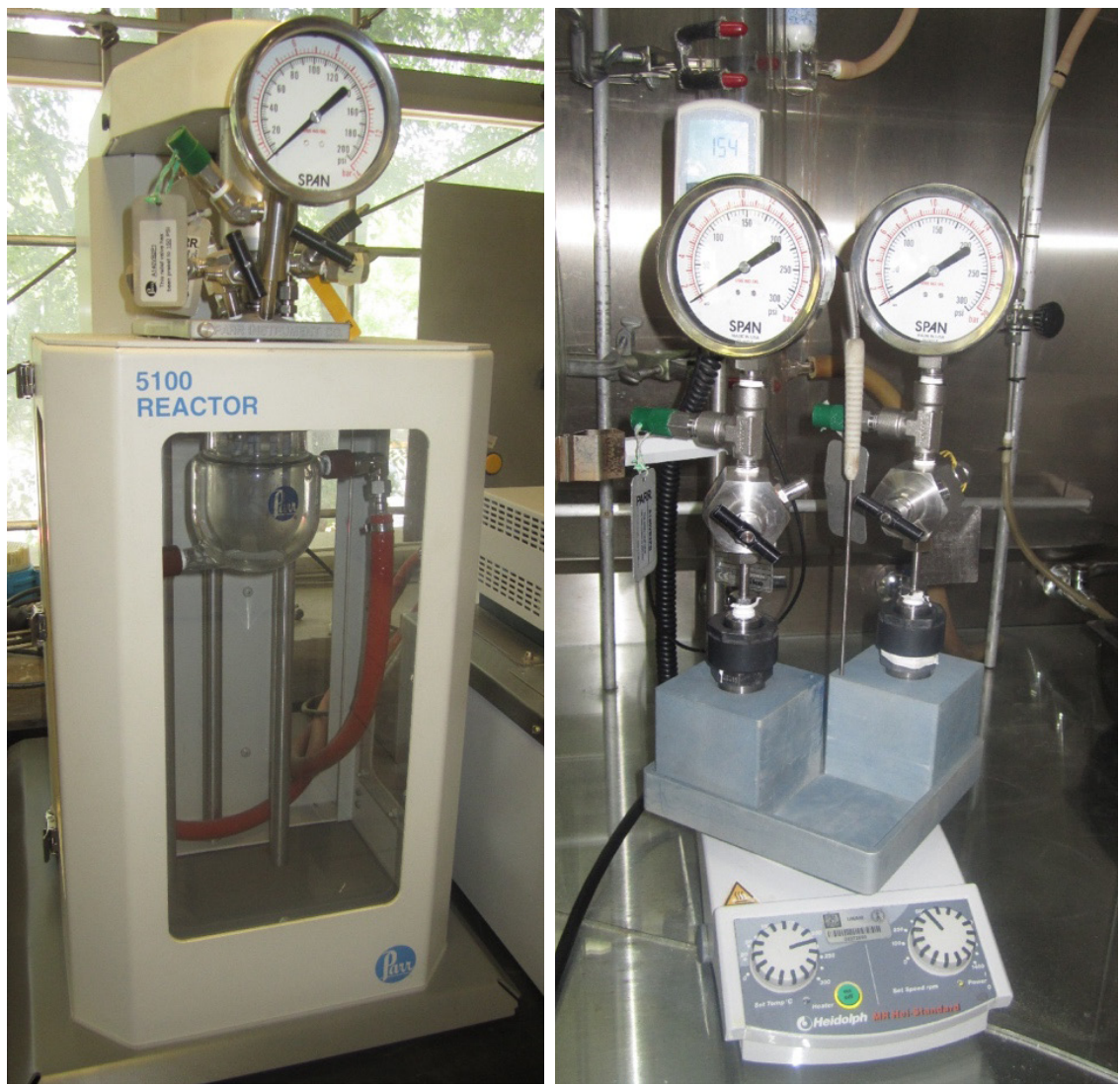

FIGURA 4. Del lado izquierdo se puede observar un reactor equipado para polimerización de etileno. A la derecha se observan dos reactores Parr para alta presión, lo que permite el estudio de reacciones de hidrogenación, carbonilación, etc. Ambos sistemas permiten el análisis y puesta a punto de las más diversas técnicas catalíticas.

Para ese mismo fin, también se cuenta con reactores para polimerización de etileno, reactores Parr de alta presión y sistemas diseñados para realizar hasta 6 reacciones de manera simultánea (figura 4), sistemas de reacción de microondas que pueden trabajar a temperatura y presión controlada (figura 5), equipos de IR y tecnología de punta en el

FIGURA 5. Horno de microondas, equipo que se utiliza para llevar a cabo reacciones con esta fuente de energía, lo que se traduce en tiempos más cortos de reacción y condiciones de química verde, este equipo soporta altas presiones y control de temperatura.

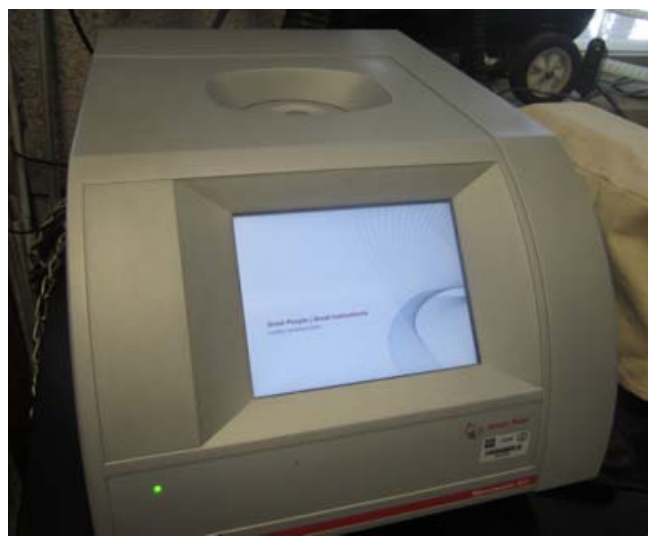




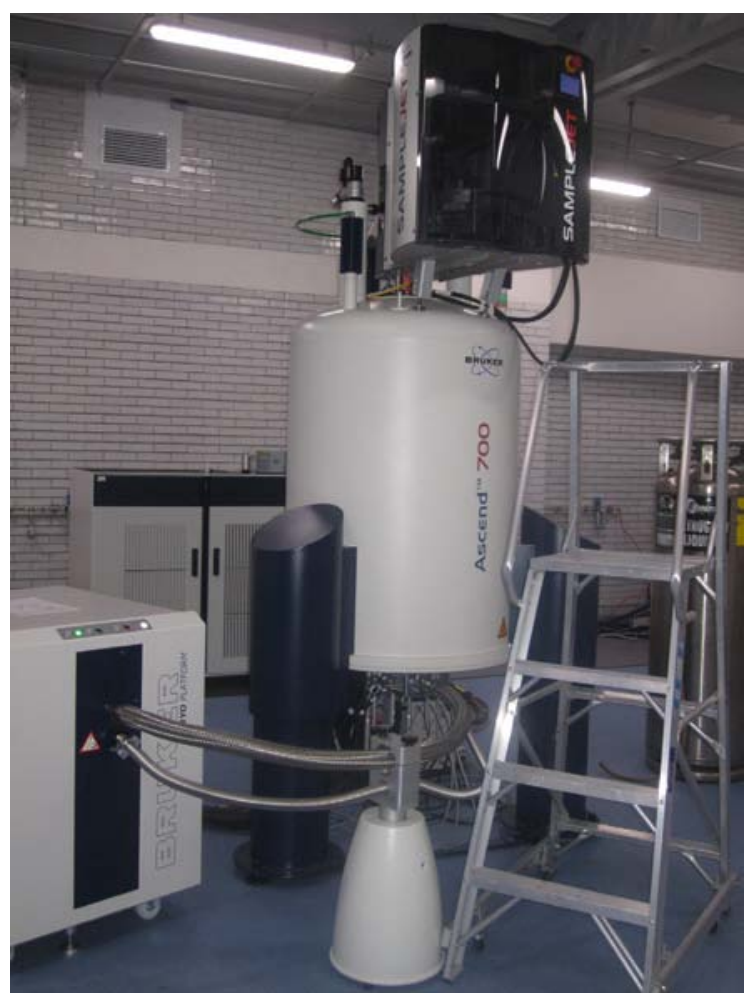

FIGURA 6. Equipo de resonancia magnética nuclear de $700 \mathrm{MHz}$ equipado con un automuestreador con capacidad mayor a las 500 muestras y con una criosonda de tres canales enfriada por helio líquido. área de resonancia magnética nuclear (300, 400, 500 y $700 \mathrm{MHz}$ ) (figura 6), uno de estos equipos de $300 \mathrm{MHz}$ se encuentra a disposición del personal del departamento de química inorgánica lo que resulta sumamente conveniente para monitorear el curso de las reacciones catalíticas. Toda esta infraestructura, junto con el enorme capital humano constituye una institución sólida y robusta con los recursos para generar investigación original y relevante en el área de catálisis homogénea.

\section{Catálisis en el Instituto de Química}

La catálisis en el IQ quizá no sea tan antigua como el área de productos naturales y química orgánica, no obstante, ha sido un área que ha crecido de manera muy importante durante las últimas cuatro décadas. A continuación, se describirán algunas de las aportaciones que dieron origen a este campo de investigación en nuestro instituto. Para hablar de los orígenes

de la investigación en el área de la catálisis, es indispensable abordar el trabajo del Dr. Jacobo Gómez Lara, quien inició formalmente sus labores en el instituto en 1963 [5] como investigador de tiempo completo. Entre otras investigaciones, el Dr. Gómez se destacó por su interés en el área de la química inorgánica que pronto se convirtió en la primera línea de investigación en el área de catálisis, con el tiempo, junto con dos alumnos suyos de posgrado, los Dres. Cecilio Álvarez y Armando Cabrera se desarrollaría la escuela de catálisis del IQ.

El primer trabajo y quizá el más importante de esa época se publicó en 1977, en esta publicación los autores estudiaron el proceso de hidroformilación catalítica del etileno en fase homogénea mediada por un compuesto organometálico de rodio (I) (1) [6], este trabajo les confiere un importante premio otorgado por la empresa Celanese Mexicana [5], con esto es justo afirmar que inicia la investigación en el área de catálisis en el IQ de la UNAM. Con el tiempo, el Dr. Gómez Lara dedicaría su vida a realizar contribuciones originales en la aplicación de compuestos de coordinación de rodio en los 
más diversos campos de la catálisis, entre otras cosas se pueden citar sus estudios sobre los efectos electrónicos y estéricos sobre el mecanismo de reacción de la decarbonilación de halogenuros de acilo mediadas por compuestos de carbonil rodio [7], y la producción de hidrógeno en medio acuoso mediante complejos catiónicos de rodio [8].

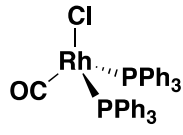

1

Con el paso del tiempo, el Dr. Cabrera fue realizando importantes contribuciones a la catálisis en México; cabe destacar su amplia labor en cuanto a procesos catalizados por cobalto, también estudió, entre otras líneas, la isomerización selectiva de epóxidos catalizada por un compuesto de coordinación de estaño de identidad $\mathrm{Sn}\left[\mathrm{Co}(\mathrm{CO})_{4}\right]_{4} ;$ [9]. Más tarde, en 2013, el grupo de investigación publicó el primer ejemplo de hidrogenación asimétrica de iminas con un sistema catalítico conformado por el dímero de tetracarbonilo de cobalto en presencia de un ligante quiral como director de la estereoselectividad de la reacción (esquema 1), [10], este tipo de procesos resultan muy atractivos para la química medicinal y la industria farmacéutica.
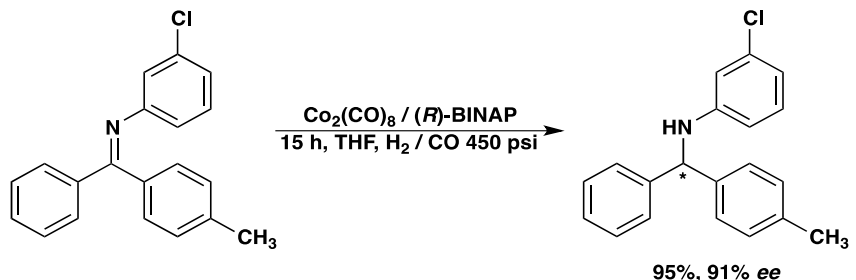

ESQUEMA 1. Primer ejemplo de hidrogenación asimétrica de iminas mediado por cobalto.

Utilizando ese mismo precursor metálico, el grupo del Dr. Cabrera estudió algunos otros procesos catalíticos, por ejemplo, la hidroformilación e hidroxialquilcarbonilación de 3,4-dihidro[2H]piranos catalizada en condiciones syngas [11]. Desde luego, en la vida de un académico tan interesado en la catálisis también se pueden encontrar otras colaboraciones con otros metales de transición, al respecto resaltan sus contribuciones sobre la carbonilación de $\alpha$-cetoalquinos mediada por níquel [12] y una excelente contribución para una reacción de aminación reductiva asimétrica de cetonas en un paso mediante el uso de un catalizador quiral de paladio (esquema 2) [13]. 


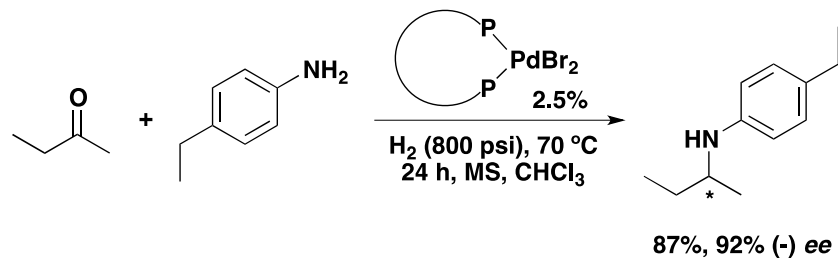

ESQUEMA 2. Aminación reductiva asimétrica de cetonas catalizada por un complejo quiral de paladio.

En el mismo terreno, el Dr. Cecilio Álvarez continuó realizando importantes colaboraciones en el área, por caso, en 2005, publicó un trabajo acerca de paladaciclos y su aplicación en la polimerización de etileno [14], en dicha publicación, los autores describieron la síntesis de un nuevo tipo de paladaciclos (2) y estudiaron su actividad catalítica para la síntesis de polietileno.

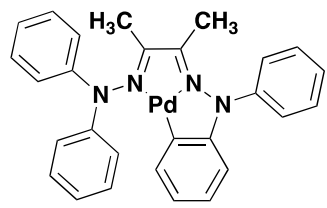

2

Así pues, a partir del trabajo de estos dos investigadores y la consolidación de sus grupos de trabajo se ha constituido la investigación en el área de catálisis como la conocemos hoy día en el IQ, en ese contexto las líneas de investigación en el área son: catálisis mediada por metales de transición, diseño y estudio de ligantes para catálisis y organocatálisis.

En general, es justo afirmar que, salvo el caso de la organocatálisis, el diseño de ligantes va de la mano con el uso del metal de transición, de manera que a continuación se describe primordialmente, la contribución que varios investigadores realizan en estas áreas de la investigación y desarrollo de sistemas catalíticos para reacciones de importancia en la química básica y aplicada.

Desde el punto de vista histórico, el uso de fosfinas como ligantes [15, 16] en reacciones catalizadas por metales de transición ha sido tan estudiado como los metales de transición en sí. Si se realizara una búsqueda en bases de datos, no sería difícil demostrar al lector que existe un número inmenso de trabajos que describen el uso de paladio, rodio, iridio, níquel, hierro, cobalto, cobre, etc., como el fragmento metálico del catalizador [17], por lo que partiremos de una breve discusión sobre la investigación que realizamos en el IQ sobre el diseño y evaluación de la actividad catalítica del binomio metal-ligante.

Con respecto a los ligantes, la versatilidad de las fosfinas y su amplia aplicabilidad en procesos catalíticos contrasta con su relativa fragilidad ante la presencia de agentes oxidantes, la humedad e incluso el medio ambiente, 
es por esta razón que una de las áreas de investigación más exploradas consiste en el diseño y desarrollo de mejores fosfinas y nuevos ligantes con y sin fósforo y con otros átomos donadores que, dependiendo de la aplicación puedan mostrar una mejora en los sistemas catalíticos actuales. En este sentido, un grupo que realiza una labor muy importante es el del Dr. David Morales, dentro de sus intereses se encuentra el desarrollo y optimización de ligantes tipo pinza [18].

Los ligantes tipo pinza constituyen una alternativa altamente rentable de estructuras polidentadas, rígidas y muy robustas. Este tipo de estructuras, debido a su versatilidad estructural, pueden ser tanto simétricas como asimétricas e incluso con centros estereogénicos (3-6) [19], lo que permite modular efectos estéricos y electrónicos que a la larga son los responsables de la actividad catalítica de un compuesto de coordinación.

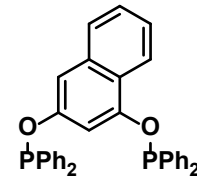

3

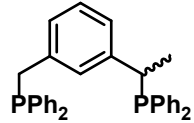

4

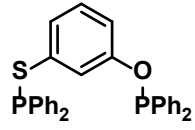

5<smiles>Pc1ccccc1Oc1cccc(-n2ccnc2P)c1</smiles>

6

Este tipo de ligantes se aplican a la investigación en los campos de la hidrogenación asimétrica, polimerización y reacciones de acoplamiento cruzado tipo Heck y Suzuki-Miyaura [19]. Al respecto, el grupo del doctor Morales ha estudiado y desarrollado la química de este tipo de ligantes, no sólo con paladio como metal sino con rutenio [19], níquel [20] e iridio [21]. En el esquema 3 se presenta la aplicación de un complejo tipo pinza de iridio con átomos de arsénico como donadores y la aplicación a reacciones de dehidrogenación catalítica.
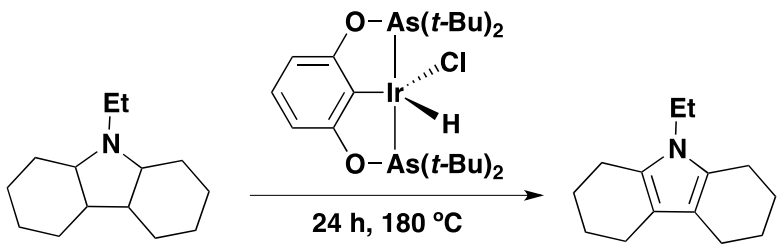

$25 \%$

ESQUEMA 3. Dehidrogenación catalítica mediada por un catalizador de iridio con ligantes tipo pinza.

Un paso más en el desarrollo de sistemas derivados de fosfinas ha sido el diseño de ligantes fósforo-nitrógeno basados en el núcleo de pirrol. Aprovechando nuestra experiencia en las reacciones de litiación de compuestos heterocíclicos, se realizó la síntesis de diversas fosfinas derivadas de pirroles 
(7) y con estos ligantes fue explorada la síntesis de los complejos de paladio correspondientes (8) (esquema 4), la actividad catalítica de estos complejos fue evaluada en reacciones de acoplamiento tipo Heck y, en vista de su buen desempeño, fueron utilizados en el paso clave de una reacción de acoplamiento intramolecular para completar la síntesis de un producto natural, la Arnotina I [ 22], el éxito del proceso evidenció la excelente actividad de este tipo de compuestos.

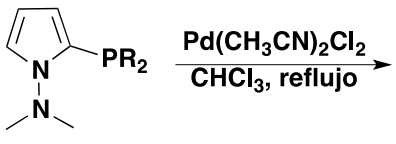

7

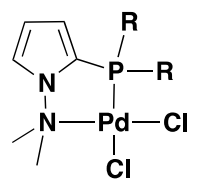

$67-88 \%$

8

ESQUEMA 4. Síntesis de los complejos 8 a partir de fosfinas derivadas de pirrol.

Animados por la síntesis de ese compuesto natural, se exploró la capacidad de estos sistemas para generar un método eficiente para las reacciones de ciclación intramoleculares mediante acoplamientos $C$ - $C$, con lo que nuestro grupo de investigación desarrolló un método catalítico en condiciones suaves para la síntesis de dibenzo- $\alpha$-pironas y las lactamas análogas [23].

Con respecto al desarrollo y aplicación de ligantes libres de fosfina, nuestro grupo de investigación ha explorado la síntesis de ligantes con diferentes esqueletos; en 2015, se describió la síntesis de una familia completa de ligantes nitrogenados (9) a partir de una reacción de Mannich sobre las correspondientes fenilhidracinas, dichos ligantes fueron evaluados en la reacción de Heck (esquema 5) [24]. Un aspecto de particular importancia es que este trabajo permitió explorar, además de la naturaleza del ligante, la activación mediante otra fuente de energía, es de particular importancia mencionar que, tanto la reacción de Mannich como la reacción de acoplamiento tipo Heck fueron promovidas irradiando las mezclas de reacción en la longitud de onda del IR, este tipo de procesos son particularmente importantes por entrar completamente dentro de lo que se conoce como química verde.

ESQUEMA 5. Reacción de Heck catalizada por paladio e irradiación IR.<smiles>[R]c1ccc(I)cc1</smiles>

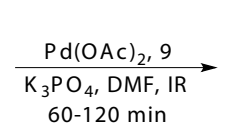<smiles>[R]OC(=O)/C=C/c1ccc([R])cc1</smiles>
$50-98 \%$

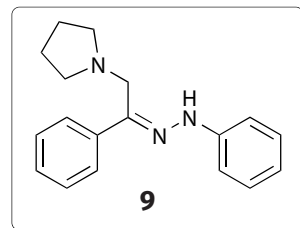


Continuando con el diseño de sistemas catalíticos, una de las aportaciones más relevantes de nuestro grupo consiste en el diseño de ligantes para reacciones de sustitución alílica y de Heck, en esta contribución se hace uso de nuestra experiencia en la síntesis de carbenos de Fischer a partir de ferroceno [25] sintetizando ferroceniltiazolinas (10) como grupos directores para la orto-litiación de ferroceno. Estos grupos fueron utilizados para agregar varios reactivos electrofílicos derivados de calcógenos, tras esta reacción fue posible la obtención de varios ligantes bidentados $(\mathbf{1 1}, \mathbf{1 2})$ que dieron excelentes resultados en las reacciones de Heck y sustitución alílica asimétricas [26]. Este tipo de ligantes ocupan un lugar sin precedente en el área de la catálisis, demostrando que es posible la sustitución con fragmento fosfina por un sulfuro y pudiendo realizar diferentes procesos catalíticos en presencia de humedad y oxígeno en condiciones suaves de reacción (figura 7).

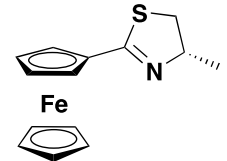

10

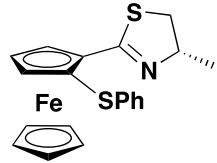

11

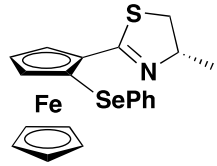

12

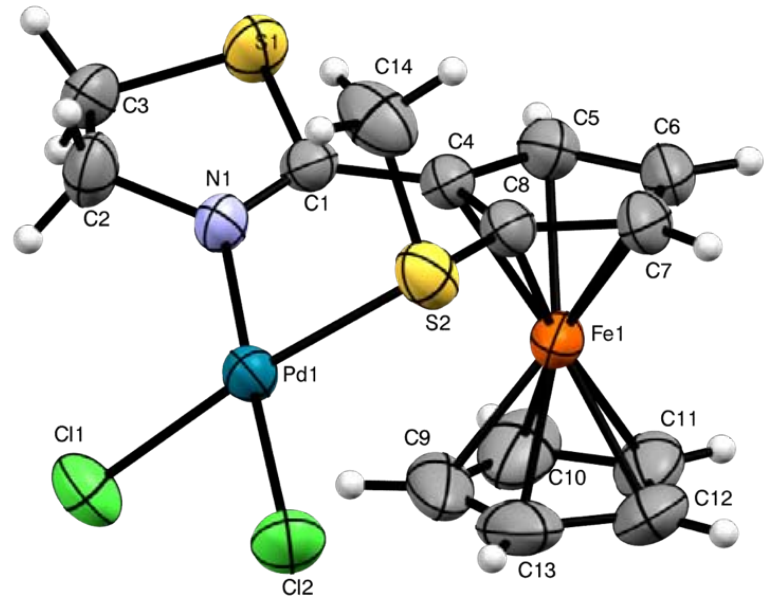

FIGURA 7. Diagrama ORTEP para un complejo de paladio utilizado para reacciones de Heck catalizadas por paladio, los elipsoides se muestran al $30 \%$ de probabilidad.

Los estudios en esta área dieron lugar a una patente [27] y ha abierto una línea de investigación completamente nueva para generar catalizadores para los más diversos procesos catalíticos, tales como hidrogenación asimétrica, Heck carbonilativo y Suzuki asimétrico, esto nos sitúa como uno de los grupos cuyos ligantes poseen uno de los más amplios intervalos de aplicabi- 
lidad, baja carga catalítica y menores tiempos de reacción lo que es altamente valioso por su potencial aplicación en procesos industriales.

Es relevante mencionar la contribución con otros metales de transición. En 2015, nuestro grupo de investigación, en colaboración con el Dr. Alvarez-Toledano, describió la síntesis de Z-enol- $\gamma$-lactonas mediante una reacción de cicloisomerización catalizada por cobre (I) en medio acuoso y microondas (esquema 6), este trabajo fue catalogado como very important paper por los editores de la revista lo que llevó al grupo de investigación a ocupar la portada de ese número [28]. Cabe aclarar que las lactonas, lactamas y derivados son compuestos muy importantes para la industria farmacéutica y la química medicinal.
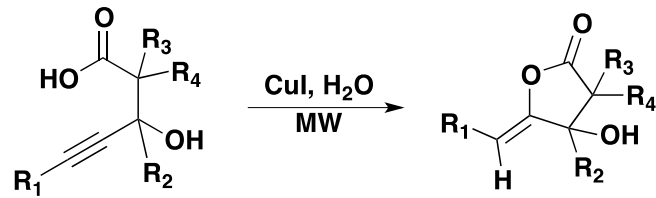

ESQUEMA 6. Síntesis de Z-enol-y-lactonas mediante una reacción catalizada por cobre (I).

Una de las principales áreas que sigue atrayendo la atención de los investigadores del IQ es el proceso de polimerización, la idea es la posibilidad de controlar el grado de polimerización, así como hacer más suaves y eficientes las condiciones experimentales. Al respecto, el desarrollo y síntesis de nuevos compuestos de coordinación de rutenio $(\mathbf{1 3}, \mathbf{1 4})$ [29] ha sido un continuo en la investigación sobre procesos catalíticos en el IQ.

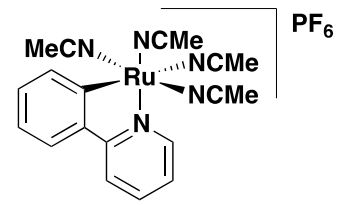

13

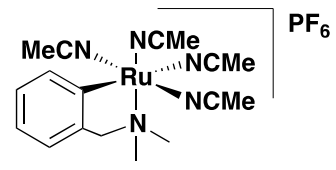

14

Es posible realizar el proceso de polimerización de acetato de vinilo de manera exitosa, utilizando microondas como fuente de energía, la reacción se lleva a cabo a $70{ }^{\circ} \mathrm{C}$ utilizando tetracloruro de carbono como iniciador y agente de transferencia de cadena. En este trabajo se ha evaluado el rol del disolvente y la fuente de calentamiento, encontrando resultados prometedores para este tipo de reacciones.

Habiendo descrito las principales líneas de investigación que se desarrollan en el IQ es necesario pensar en el futuro de la investigación en este campo, como una de las estrategias importantes para seguir explorando líneas de investigación novedosas, que vayan de acuerdo con el desarrollo que la industria y la ciencia demandan; en este sentido uno de los proyectos im- 
portantes es la incorporación de doctores jóvenes a la planta académica del instituto, que permitirán enriquecer y nutrir la investigación en el área.

En este contexto, uno de los investigadores que se incorporaron recientemente al IQ en el área de catálisis es el Dr. Amézquita-Valencia quien, en 2016, publicó un proceso catalítico Ligand Free sin precedentes, utilizando acetato de paladio en presencia de una base para la síntesis de anilinas vía una reacción de transposición de grupo bencilo, este tipo de procesos son muy poco comúnes lo que ha valido que este trabajo [30] se haya publicado como hot paper en una importante revista del medio. Entre los intereses del Dr. Amézquita se encuentra la catálisis asimétrica mediada por paladio. Finalmente, la Dra. Ana Sofía Varela Gasque, quien se incorporó recientemente a nuestro instituto, realiza investigación enfocada en la activación de $\mathrm{CO}_{2}$, [31, 32], y los procesos de electrocatálisis por medio de sistemas nanoestructurados [33].

Por último, dentro del IQ, en el Departamento de Química Orgánica también se cultivan otras áreas de la catálisis, como la organocatálisis, este tipo de química no se basa en el uso de metales de transición o ligantes quirales, sino en interacciones que hacen posible un reconocimiento entre moléculas generando un arreglo supramolecular que sirve para dirigir la reactividad en una transformación específica. Al respecto, el principal líder de este tipo de investigación en el IQ es el Dr. Marcos Hernández, cuya línea de investigación se basa en el uso de ureas [34] y tioureas quirales [35] $(\mathbf{1 5}, \mathbf{1 6})$ para inducir transformaciones catalíticas sobre sustratos orgánicos. Este tipo de catalizadores actúan formando puentes de hidrógeno, realizando un reconocimiento molecular de estructuras potencialmente aniónicas para facilitar transformaciones quirales y resoluciones cinéticas.

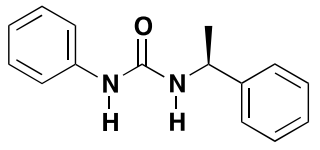

15

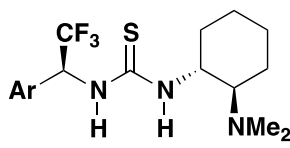

16

En general se ha tenido éxito en aplicar este tipo de sistemas a reacciones de adición tipo Michael de la 2,4-pentanodiona a $\beta$-nitroestireno (esquema 7); la química supramolecular y en particular los procesos catalíticos que comprenden este tipo de principios son un tema de actualidad en cuanto a la catálisis quiral e implica un nicho de oportunidad para hacer investigación básica y aplicada en el área.

En general, se han presentado las principales líneas de investigación que desarrolla el IQ en cuanto a catálisis, cabe aclarar que esta área tiene el más profundo impacto en todas las demás áreas de la química, por ejemplo, en la síntesis de fármacos, síntesis total, síntesis de dispositivos optoelectrónicos, 

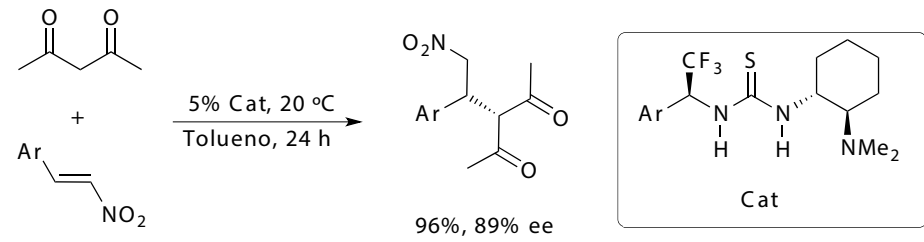

ESQUEMA 7. Reacción de Michael asimétrica mediante el uso de un derivado quiral de tiourea.

máquinas moleculares, etc. Los métodos que se desarrollan en el IQ alcanzan, en resumen, todas las áreas de la química aplicada.

\section{Conclusiones}

El Instituto de Química de la UNAM es una institución con 75 años de renombre y con una sólida tradición de realizar química en nuestro país, este prestigio hace que cuente con los recursos tecnológicos, materiales y humanos para realizar investigación de punta en el área de la catálisis. La institución cuenta con un nutrido grupo de expertos que están encargados en dirigir los esfuerzos individuales y colectivos hacia el desarrollo de metodologías limpias, económicas y adecuadas para el desarrollo de la sociedad y de todos los actores que participan de ella. Por otro lado, el IQ posee los más amplios convenios de colaboración con otras entidades a nivel mundial [15], basta mencionar entre otros, la Universidad de Harvard, el MIT, el CNRS, la Universidad Paul Sabatier (Universidad de Toulouse III) y, por supuesto, ocupa un lugar predominante en colaboraciones con instituciones a nivel nacional, como por ejemplo la Secretaría de Energía, el Consejo Nacional de Ciencia y Tecnología, la Universidad Autónoma del Estado de México con quien se ha construido el Centro Conjunto de Investigación en Química Sustentable, la Universidad Nacional Autónoma del Estado de Morelos, entre otros, por mencionar parte de la inmensa red de colaboración con la que cuenta el IQ para realizar su labor.

Nuestro grupo de investigación, por ejemplo, ha establecido una estrecha relación con investigadores de prestigiosas universidades en Chile y Alemania a través del núcleo milenio, enfocado en impulsar desarrollos catalíticos orientados a resolver procesos industriales en el área de polímeros biodegradables. Actualmente forma parte de un Laboratorio Virtual conformado por diferentes dependencias de la UNAM y dos instituciones francesas, en este laboratorio internacional asociado se desarrolla investigación conjunta para resolver problemas en el área de catálisis enfocando su empleo en el desarrollo de nuevos materiales moleculares con posible aplicación en la ciencia de materiales.

La capacidad del Instituto en cuanto a infraestructura y convenios de colaboración le permiten ser una institución participante en varios programas de posgrado en la universidad, lo que permite que sea participe de la forma- 
ción de innumerables recursos humanos en los niveles de licenciatura, maestría, doctorado y un lugar sumamente cotizado para realizar estancias postdoctorales. Todo esto permite afirmar que el IQ de la UNAM debe ser considerado uno de los mejores sitios en México para hacer ciencia y una dependencia que puede brindar lazos de colaboración para una infinidad de proyectos en las más diversas áreas de la ciencia

\section{Referencias}

[1] L. Miramontes, P. Aguinaco, M. A. Romero. (1960). J. Am. Chem. Soc., 82, 6153.

[2] T. Ríos, F. G. Gómez. (1969). Tetrahedron Lett., 10, 2929.

[3] A. Rodríguez-Romero, K. G. Ravichandran, M. Soriano-García. (1991). FEBS Letters, 291, 307.

[4] J. Peón. Informe de Actividades, Instituto de Química-UNAM, 2014-2015. <http:// www.iquimica.unam.mx/images/documentos/INFORME_2014-2015.pdf>.

[5] C. Álvarez-Toledano. (2014). Aportes recientes a la historia de la química en México: Departamento de Química Inorgánica, Eds. M. P. Ramos-Lara, F. LeónOlivares (eds.). Ciencia y Tecnología en la Historia de México. México.

[6] N. Rosas, J. Gómez-Lara, A. Cabrera, C. Álvarez. (1977). Revista Latinoamericana de Química, 8, 121.

[7] F. Delgado, A. Cabrera, J. Gómez-Lara. (1983). J. Molecular Catal., 22, 83.

[8] A. Cabrera, J. Gómez-Lara, M. Alcaraz. (1989). New J. Chem., 13, 103.

[9] A. Cabrera, F. Mathe, Y. Castanet, A. Mortreux, F. Petit. (1991). J. Mol. Catal., 64, L11.

[10] M. Amezquita-Valencia, A. Cabrera. (2013). J. Mol. Catal. A: Chemical, 366, 17.

[11] J. L. Arias, P. Sharma, A. Cabrera, F. Beristain, R. Sampere, C. Arizmendi. (2013). Transition Met. Chem., 38, 787.

[12] H. Arzoumanian, M. Jean, D. Nuel, A. Cabrera, J. L. G. Gutierrez, N. Rosas. (1995). Organometallics, 14, 5438.

[13] L. Rubio-Pérez, F. Pérez-Flores, P. Sharma, L. Velasco, A. Cabrera. (2009). Org. Lett., 11, 265.

[14] M. A. Pérez, R. Quijada, F. Ortega-Jiménez, C. Álvarez-Toledano. (2005). J. Mol. Catal. A: Chemical, 226, 291.

[15] H. Fernández-Pérez, P. Etayo. A. Panossian, A. Vidal-Ferran. (2011). Chem. Rev., 111, 2119.

[16] P. Ruiz-Castillo, S. L. Buchwald. (2016). Chem. Rev., 116, 12564.

[17] <http://scifinder.cas.org >.

[18] D. Morales-Morales. (2007). D. Morales-Morales, C. M. Jensen (EDS.), The Chemistry of Pincer Compounds, Elsevier, Amsterdam, ISBN-13: 978-0-444-53138-4.

[19] M. Asay, D. Morales-Morales. (2015). Dalton Trans., 44, 17432.

[20] M. Basauri-Molina, S. Hernández-Ortega, D. Morales-Morales. (2014). Eur. J. Inorg. Chem., 4619.

[21] D. F. Brayton, P. R. Beaumont, E. Y. Fukushima, H. T. Sartain, D. Morales-Morales, C. M. Jensen. (2014). Organometallics, 33, 5198. 
[22] J. V. Suárez-Meneses, E. Bonilla-Reyes, E. A. Blé-González, M. C. Ortega-Alfaro, R. A. Toscano, A. Cordero-Vargas, J. G. López-Cortés. (2014). Tetrahedron, 70, 1422.

[23] J. V. Suárez-Meneses, A. Oukhrib, M. Gouygou, M. Urrutigoity, J.-C. Daran, A. Cordero-Vargas, M. C. Ortega-Alfaro, J. G. López-Cortés. (2016). Dalton Trans., 45, 9621.

[24] F. Ortega-Jiménez, J. G. Penieres-Carrillo, S. Lagunas-Rivera, J. G. López-Cortés, C. Álvarez-Toledano, M. C. Ortega-Alfaro. (2015). RSC Adv., 5, 80911.

[25] J. G. López-Cortés, L. F. Contreras de la Cruz, M. C. Ortega-Alfaro, R. A. Toscano, C. Álvarez-Toledano, H. Rudler. (2005). J. Organomet. Chem., 690, 2229.

[26 a] E. P. Sanchez-Rodríguez, F. Hochberger-Roa, R. Corona-Sánchez, J. E. Barquera-Lozada, R. A. Toscano, M. Urrutigoïty, M. Gouygou, M. C. Ortega-Alfaro, J. G. López-Cortés. (2017). Dalton Trans., 46, 1510; [ 26 b] R. Corona-Sánchez, R. A. Toscano, M. C. Ortega-Alfaro, C. Sandoval-Sánchez, J. G. López-Cortés. (2013). Dalton Trans., 42, 11992.

[27] F. F. K. Hochberger-Roa, J. G. López-Cortés. (2016). Expediente de Patente: MX/a/2015/016922, México.

[28] M. E. López-Reyes, R. A: Toscano, J. G. López-Cortés, C. Álvarez-Toledano. (2015). Asian J. Org. Chem., 4, 545.

[29] J. Olvera-Mancilla, S. López-Morales, J. Palacios-Alquisira, D. Morales-Morales, R. Le Lagadec, L. Alexandrova. (2014). Polymer, 55, 1656.

[30] M. Amézquita-Valencia, H. Alper. (2016). Chem. Eur. J., 22, 16774.

[31] H. Mistry, A. S. Varela, C. S. Bonifacio, I. Zegkinoglou, I. Sinev, Y.-W. Choi, K. Kisslinger, E. A. Stach, J. Yang, P. Strasser. (2016). Nature Commun., 7, 12123.

[32] A. S. Varela, W. Ju, T. Reier, P. Strasser. (2016). ACS Catal., 6, 2136.

[33] H. Mistry, A. S. Varela, S. Kühl, P. Strasser, B. Roldan-Cuenya. (2016). Nature Rev. Mater., 1, 16009.

[34] M. Cortés-Hernández, S. Rojas-Lima, M. Hernández-Rodríguez, J. Cruz-Borbolla, H. López-Ruiz. (2016). Helv. Chim. Acta, 99, 416.

[35] E. I. Jiménez, W. E. Vallejo-Narváez, C. A. Román-Chavarría, J. VÁzquez-Chávez, T. Rocha-Rinza, M. Hernández-Rodríguez. (2016). J. Org. Chem., 81, 7419. 